\title{
Segurança e qualidade do cuidado em saúde na perspectiva do usuário: estudo exploratório de grupos do Facebook
}

\author{
Safety and quality of healthcare from the user perspective: exploratory \\ study of Facebook groups
}

\section{Seguridad y calidad de la atención de salud desde la perspectiva del usuario: estudio exploratorio de grupos del Facebook}

Vanessa Cristina Felippe Lopes Villar ${ }^{1, a}$

vanessalopesvillar@gmail.com | https://orcid.org/o0oo-0002-4469-2796

Elaine Teixeira Rabello ${ }^{2, b}$

e.rabello@kit.nl | https://orcid.org/oooo-0002-8324-1453

\section{Mônica Martins $3, c$}

martins@ensp.fiocruz.br | https://orcid.org/o000-0002-9962-0618

\footnotetext{
${ }^{1}$ Fundação Oswaldo Cruz, Instituto Nacional de Infectologia Evandro Chagas. Rio de Janeiro, RJ, Brasil.

2 Royal Tropical Institute. Amsterdam, Holanda.

${ }^{3}$ Fundação Oswaldo Cruz, Escola Nacional de Saúde Pública Sergio Arouca. Rio de Janeiro, RJ, Brasil.

a Doutorado em Saúde Pública pela Fundação Oswaldo Cruz.

b Ph. D em Santé Publique pela Université de Montréal.

c Doutorado em Saúde Coletiva pelo Universidade do Estado do Rio de Janeiro.
}

\section{RESUMO}

O objetivo do estudo apresentado neste artigo é discutir segurança e qualidade do cuidado em saúde, usando o conteúdo compartilhado em grupos do Facebook e refletir sobre a perspectiva do paciente quanto ao tema. Trata-se de um estudo qualitativo que utilizou, como fonte de dados, postagens de três grupos que abordavam a temática na plataforma de mídia digital Facebook por ocasião da pesquisa. Para analisar os dados, foi utilizado o software Iramutec e realizada a análise de conteúdo temática. Emergiram quatro categorias: incidentes e eventos adversos; fatores contribuintes; consequências dos eventos adversos; reparação ou mitigação desses incidentes e eventos adversos. Os incidentes e eventos adversos foram descritos pelos usuários dos grupos do Facebook como 'erros médicos'. Apesar da relevância para a vida de pacientes e familiares que passaram por incidentes e eventos adversos, o conteúdo das postagens sobre o que ocorreu é pouco compartilhado, o que parece indicar que os grupos funcionam como um portal de notícias da mídia tradicional.

Palavras-chave: Segurança do paciente; Qualidade da assistência à saúde; Internet; Mídias sociais; Facebook. 


\title{
ABSTRACT
}

The aim of the study presented in this article is to discuss the safety and quality of health care using content shared in Facebook groups and to reflect on patient's perspective on these issues. It is a qualitative study using as data source posts from three groups that addressed these issues on Facebook during the research period. Data analysis applied the Iramutec software, and it was based on thematic content analysis. Four categories emerged: incidents and adverse events; contributing factors; consequences of adverse events; reparation or mitigation of these incidents and adverse events. The users of Facebook groups described incidents and adverse events as 'medical errors'. Despite the relevance to patients and family members who experienced adverse incidents and adverse events, the content of the posts regarding the events is rarely shared, which seems to indicate that these groups have been functioning as news portal of mainstream media.

Keywords: Patient safety; Quality of health care; Internet; Social media; Facebook.

\section{RESUMEN}

El objetivo del estudio presentado en este artículo es debatir la seguridad y la calidad de la atención de salud a través de los contenidos compartidos en los grupos de Facebook y reflexionar sobre la perspectiva del paciente acerca del tema. Él constituye un estudio cualitativo que utilizó como fuente de datos las publicaciones de tres grupos que abordaron el tema en la plataforma de medios de comunicación digitales Facebook. El análisis de datos se hizo con el programa informático Iramutec y se basó en el análisis de contenido temático. Surgieron cuatro categorías: incidentes y eventos adversos, factores contribuyentes, consecuencias de los eventos adversos, reparación o mitigación de estos incidentes y eventos adversos. Los usuarios de los grupos del Facebook describieron los incidentes y eventos adversos como 'errores médicos'. A pesar de la relevancia para la vida de los pacientes y familiares que experimentaron incidentes y eventos adversos, el contenido es poco compartido, lo que parece indicar que los grupos funcionan como un portal de noticias de los medios de comunicación dominantes.

Palabras clave: Seguridad del paciente; Calidad de la atención de salud; Internet; Medios de comunicación sociales; Facebook.

\begin{abstract}
Contribuição dos autores:
Concepção e desenho do estudo: Vanessa Cristina F. L. Villar; Elaine T. Rabello.

Aquisição, análise ou interpretação dos dados: Vanessa Cristina F. L. Villar.

Redação do manuscrito: Vanessa Cristina F. L. Villar; Elaine T. Rabello; Mônica Martins.

Revisão crítica do conteúdo intelectual: Vanessa Cristina F. L. Villar; Elaine Rabello; Mônica Martins.
\end{abstract}

Declaração de conflito de interesses: não há.

Fontes de financiamento: não houve.

Considerações éticas: a pesquisa foi aprovada pelo Comitê de Ética em Pesquisa da Escola Nacional de Saúde Pública Sérgio Arouca com parecer número 3.492.606, de acordo com a Resolução CNS/MS n 466/2012.

Agradecimentos/Contribuições adicionais: Agradecimento ao Conselho Nacional de Desenvolvimento Científico e
Tecnológico ao CNPq pela bolsa de produtividade outorgada a MM (PQ 306100/2019-3).

Histórico do artigo: submetido: 18 jun. 2021 | aceito: 12 nov. 2021 | publicado: 25 fev. 2022.

Apresentação anterior: não há.

Licença CC BY-NC atribuição não comercial. Com essa licença é permitido acessar, baixar (download), copiar, imprimir, compartilhar, reutilizar e distribuir os artigos, desde que para uso não comercial e com a citação da fonte, conferindo os devidos créditos de autoria e menção à Reciis. Nesses casos, nenhuma permissão é necessária por parte dos autores ou dos editores. 


\section{INTRODUÇÃO}

O engajamento e empoderamento dos pacientes na segurança e qualidade do cuidado vêm sendo reconhecidos como uma importante estratégia para o aprendizado e a melhoria da prestação do cuidado em saúde (WHO, 2013). Os pacientes atribuem uma perspectiva sobre segurança distinta ou complementar aos profissionais de saúde, embora haja diferença nos mecanismos de detecção de incidentes e eventos adversos no cuidado em saúde especificamente voltados para esses sujeitos. Deste modo, a perspectiva do paciente emerge, também, com o potencial de ajudar a construir novos processos de cuidado e a melhorar o desempenho de equipes clínicas e organizações de saúde (O'HARA et al., 2018).

O engajamento dos pacientes na segurança e qualidade do cuidado pode ocorrer de diversas maneiras; tradicionalmente, a mais comum é o relato ou a notificação de incidentes e eventos adversos. Todavia, novas maneiras de fazê-lo surgem com o avanço da internet e o uso de novas tecnologias de informação para saúde (DESLANDES; COUTINHO, 2020; HUERTA et al., 2016).

As plataformas de mídia digital surgem como alternativas aos modos tradicionais de comunicação entre pacientes e profissionais, e são cada vez mais acessadas pelos pacientes e usuários de serviços de saúde (DESLANDES; COUTINHO, 2020; HUERTA et al., 2016; ROZENBLUM; BATES, 2017). Diversas dessas vêm sendo utilizadas pelos pacientes para comunicar-se com profissionais de saúde ou com outros usuários, externar suas preocupações sobre saúde, compartilhar experiências, obter informações ou avaliar serviços de saúde (GREAVES et al., 2013; VAN DE BELT et al., 2015; ROZENBLUM; BATES, 2013). Esse conjunto de informações gerado nas interações e registrado nas plataformas é descrito na literatura como "nuvem da experiência do paciente" (GREAVES et al., 2013, p. 251).

Dentre as plataformas de mídia digital, destaca-se o Facebook, que possui o maior número de usuários no mundo e tem grande abrangência no Brasil, contando com mais de 136 milhões de contas ativas em 2019 (STATISTA RESEARCH DEPARTMENT, 2021). Servindo de espaço para exteriorização de preocupações sobre segurança do paciente e qualidade do cuidado, compartilhamento de experiências, pedidos de indicações profissionais ou ajuda, essa plataforma revelou-se, assim, nos últimos anos, uma importante ferramenta e fonte de dados para pesquisa qualitativa (FRANZ et al., 2019).

Tendo em vista a importância crescente do Facebook nos últimos anos como meio de compartilhamento de informações sobre o cuidado em saúde (FRANZ et al., 2019), mobilização social e troca de experiências (MIRANDA; ROCHA, 2018), faltam estudos que abordem o uso dessa plataforma de mídia digital por usuários para discutir segurança do paciente e qualidade do cuidado. Este estudo busca preencher essa lacuna e, para isso, discutirá essas questões usando o conteúdo compartilhado em grupos do Facebook, de modo a refletir sobre a perspectiva do paciente em relação ao tema.

\section{MÉTODO}

Trata-se de um estudo qualitativo que utilizou a plataforma de mídia digital Facebook como fonte de dados. Por meio dessa plataforma é possível gerar uma quantidade de dados ou conteúdos, que podem ser textos, imagens, vídeos e reações. Existem três tipos de dados textuais gerados no Facebook: (i) postagens escritas pelo usuário ou que aparecem da linha de tempo de outro usuário; (ii) comentários, que são respostas às postagens; e (iii) mensagens enviadas de forma privada a outro usuário, em geral um amigo (FRANZ et al., 2019).

Métodos de pesquisa qualitativa em mídias sociais como o Facebook podem ser descritos de três maneiras: (i) análise ativa, que envolve a comunicação dos pesquisadores com os usuários; (ii) análise passiva, que envolve o estudo de padrões de informação ou de interações entre os usuários dos grupos do Facebook; e (iii) pesquisa por meio de autoidentificação, ou seja, quando os pesquisadores usam, por exemplo, 
o Facebook como ferramenta de recrutamento para entrevistas, grupos focais ou enquetes realizadas pela internet (FRANZ et al., 2019). Para fins deste trabalho, que explora a perspectiva da segurança do paciente e a qualidade do cuidado nos grupos de Facebook, optou-se pela análise passiva com a postura de 'lurker' (observador), ou seja, coleta de dados sem identificação dos pesquisadores nos grupos estudados.

A coleta de dados foi realizada em grupos de Facebook selecionados nos meses de setembro e outubro de 2019. A escolha do período se deu considerando que 17 de setembro foi o primeiro Dia Mundial da Segurança do Paciente (WHO, 2019), estendeu-se até o mês de outubro no qual é comemorado o dia do médico no Brasil (18 de outubro).

\section{Procedimentos de coleta}

Com navegador e perfis neutros, foi inserido no buscador do Facebook a expressão 'erro médico'. Ela foi escolhida após teste prévio das expressões: 'segurança do paciente'; 'qualidade na saúde’; 'problemas no cuidado em saúde' e 'erro médico'.

As expressões 'segurança do paciente' e 'qualidade do cuidado' revelaram páginas e grupos voltados para os profissionais de saúde, enquanto a expressão 'problemas no cuidado em saúde' estava voltada para o público em geral; porém, em todas havia conteúdo predominante de divulgação de cursos, eventos e serviços. Assim, a expressão 'erro médico' mostrou-se mais adequada para tratar as questões de segurança do paciente e qualidade do cuidado dentro do objetivo deste estudo, pois revelou grupos com postagens de perfis e páginas administradas por usuários aparentemente leigos e que tratavam de incidentes e eventos adversos no cuidado em saúde.

Foram selecionados grupos públicos utilizando a ferramenta 'filtrar resultados', que resultou em 69 grupos. Para a coleta de dados, foram selecionados os três grupos com maior número de usuários e maior número de postagens diárias (Tabela 1).

\section{Tabela 1 - Número de usuários e de postagens por grupo selecionado no Facebook, no período entre setembro e outubro de 2019}

\begin{tabular}{|ccc}
$\begin{array}{c}\text { Identificação } \\
\text { do grupo }\end{array}$ & $\begin{array}{c}\text { Número } \\
\text { de usuários }\end{array}$ & $\begin{array}{c}\text { Número } \\
\text { de postagens }\end{array}$ \\
\hline Grupo 1 & 741 & 801 \\
Grupo 2 & 1.100 & 717 \\
Grupo 3 & 1.300 & 91 \\
\hline
\end{tabular}

Fonte: elaboração das autoras.

Apesar de se tratar de grupos públicos, neste estudo foi realizado um procedimento de não identificação dos grupos, usuários e estabelecimentos de saúde para resguardar o seu anonimato. Estabeleceram-se códigos para grupos e postagens, identificando nomes dos pacientes e familiares apenas com a inicial e suprimindo nomes de estabelecimentos.

A coleta de dados foi manual com print de página, colagem das imagens, textos, anotação das curtidas e comentários. Dos três grupos selecionados, foram coletadas um total de 1.609 postagens, que foram lidas na íntegra e arquivadas. A análise iniciou-se pari passu à coleta, que foi suspensa quando houve saturação de dados, ou seja, no momento em que não apareceram categorias novas a serem analisadas. 


\section{Tratamento e análise dos dados}

Na primeira etapa de tratamento manual foram excluídas as duplicatas, os vídeos, as lives e postagens relacionadas a (i) problemas no cuidado em saúde ocorridos no exterior; (ii) propagandas e chamadas para programas de televisão; (iii) problemas relacionados à formação de médicos, (iv) exercício ilegal da profissão; (v) violência urbana envolvendo médicos, entre outros. No caso de postagem duplicada, foi eleita a mais antiga para análise, com maior número de reações, comentários e compartilhamentos. Para a análise foram incluídas (i) as postagens dos pacientes que abordam os problemas no cuidado de saúde na perspectiva do paciente, (ii) as postagens referentes a matérias de jornais sobre esse tema.

Na segunda etapa, foi realizada a análise de conteúdo lexical do texto, utilizando o software Iramutec. A análise textual provida pela ferramenta consiste em um tipo específico de análise de dados de material transcrito de entrevistas ou publicado na forma de textos (NASCIMENTO; MENANDRO, 2006). Para este estudo, foi realizada a análise de similitude, que possibilita identificar as ocorrências da ligação entre diversas palavras em cada grupo de usuários, de modo a auxiliar a identificação da estrutura de um corpus textual (CAMARGO; JUSTO, 2013).

$\mathrm{Na}$ terceira etapa, as postagens foram lidas e categorizadas segundo seu conteúdo, seguindo os procedimentos de análise de conteúdo temática (BARDIN, 2011). Deste processo, emergiram quatro categorias, as duas primeiras relacionadas à International classification for patient safety (ICPS) (WHO, 2009): (i) incidentes e eventos adversos, (ii) fatores contribuintes; e as outras duas, complementares: (iii) consequências dos eventos adversos, e (iv) reparação ou mitigação dos incidentes e eventos adversos (Quadro 1). Em seguida, as subcategorias foram reagrupadas de acordo com o conteúdo, dando origem a 20 subcategorias.

Quadro 1 - Categorias e subcategorias relacionadas às postagens nos grupos do Facebook selecionados

\begin{tabular}{|c|c|c|c|c|}
\hline & Categoria* & Subcategoria & Exemplo & $(n)$ \\
\hline \multirow[t]{8}{*}{1} & $\begin{array}{l}\text { Incidentes e } \\
\text { eventos adversos }\end{array}$ & $\begin{array}{l}\text { Processos administrativos } \\
\text { indiretos relacionados ao } \\
\text { cuidado }\end{array}$ & $\begin{array}{l}\text { Atraso no agendamento ou realização de consulta, } \\
\text { exames, tratamento, diagnóstico ou cirurgia }\end{array}$ & 72 \\
\hline & & Cirurgia & $\begin{array}{l}\text { Esquecimento de corpo estranho, infecções pós- } \\
\text { cirúrgicas, controle da dor pós-operatória }\end{array}$ & 40 \\
\hline & & $\begin{array}{l}\text { Procedimento e } \\
\text { processo clínico }\end{array}$ & $\begin{array}{l}\text { Erro no diagnóstico, tratamento inadequado, } \\
\text { falta de atendimento, atendimento deficiente e } \\
\text { imperícia }\end{array}$ & 34 \\
\hline & & Comportamento & $\begin{array}{l}\text { Abuso, violência, estupro e assédio sexual no } \\
\text { ambiente do cuidado em saúde. Violência entre } \\
\text { paciente - profissional, profissional-paciente e } \\
\text { entre profissionais }\end{array}$ & 25 \\
\hline & & Medicamentos & $\begin{array}{l}\text { Erro de prescrição, troca de medicamentos, } \\
\text { reações alérgicas, reações adversas e reação no } \\
\text { local da aplicação }\end{array}$ & 14 \\
\hline & & Infecções & Infecção, hospitalar e sepse neonatal & 11 \\
\hline & & $\begin{array}{l}\text { Procedimentos } \\
\text { não cirúrgicos }\end{array}$ & $\begin{array}{l}\text { Troca de curativos, punção para administração de } \\
\text { medicamentos }\end{array}$ & 7 \\
\hline & & $\begin{array}{l}\text { Exames laboratoriais e } \\
\text { de imagem }\end{array}$ & $\begin{array}{l}\text { Troca de exames, erro na realização de exame, } \\
\text { falsificação de exames, não realização de exames } \\
\text { prescritos }\end{array}$ & 6 \\
\hline
\end{tabular}


(conclusão)

\begin{tabular}{|c|c|c|c|c|}
\hline & Categoria* & Subcategoria & Exemplo & (n) \\
\hline & & $\begin{array}{l}\text { Queda } \\
\text { Reações à transfusão de } \\
\text { sangue } \\
\text { Erro médico }\end{array}$ & $\begin{array}{l}\text { Queda do berço e na ambulância } \\
\text { Troca de bolsas de sangue } \\
\text { Erro ou problema ocorrido na prática do cuidado } \\
\text { não necessariamente cometido pelo profissional } \\
\text { médico }\end{array}$ & $\begin{array}{l}4 \\
1 \\
12\end{array}$ \\
\hline 2 & $\begin{array}{l}\text { Fatores } \\
\text { contribuintes }\end{array}$ & $\begin{array}{l}\text { Ambiente e estrutura } \\
\text { Negligência } \\
\text { Comunicação } \\
\text { Profissionais } \\
\text { Identificação do paciente }\end{array}$ & $\begin{array}{l}\text { Problemas relacionados ao funcionamento da } \\
\text { ambulância, ar-condicionado, incêndios, falta } \\
\text { de equipamentos, insumos e medicamentos, } \\
\text { superlotação/falta de leitos, e reaproveitamento de } \\
\text { material de uso único } \\
\text { Ausência do cuidado, cuidado displicente } \\
\text { Problemas relacionados à comunicação } \\
\text { profissional-paciente e prontuários } \\
\text { Falta de profissionais, abandono de posto de } \\
\text { trabalho e omissão de socorro } \\
\text { Identificação de recém-nascido e desaparecimento } \\
\text { de corpo }\end{array}$ & $\begin{array}{l}33 \\
25 \\
22 \\
7\end{array}$ \\
\hline 3 & $\begin{array}{l}\text { Consequências dos } \\
\text { eventos adversos }\end{array}$ & $\begin{array}{l}\text { Óbitos } \\
\text { Incapacidade temporária ou } \\
\text { permanente } \\
\text { Problemas emocionais }\end{array}$ & $\begin{array}{l}\text { Óbitos ocorridos no cuidado hospitalar ou após } \\
\text { alta, investigação de óbitos } \\
\text { Perda parcial ou total de órgão ou função } \\
\text { temporária ou permanente } \\
\text { Depressão, medo, tristeza, decepção, desânimo }\end{array}$ & $\begin{array}{l}77 \\
17 \\
14\end{array}$ \\
\hline 4 & $\begin{array}{l}\text { Reparação ou } \\
\text { mitigação dos } \\
\text { eventos adversos }\end{array}$ & Judicialização & $\begin{array}{l}\text { Processos judiciais de reparação de danos } \\
\text { materiais ou morais }\end{array}$ & 58 \\
\hline
\end{tabular}

*Em algumas postagens foram encontradas mais de uma categoria.

Fonte: elaboração das autoras, baseado em dados retirados do Facebook.

Em seguida, as subcategorias foram reagrupadas de acordo com o conteúdo, dando origem a 19 subcategorias.

\section{RESULTADOS E DISCUSSÃO}

Foram analisadas 299 postagens subdivididas em: (i) reportagens $(n=254)$ e (ii) postagens dos usuários e administradores, nas quais manifestam suas impressões, queixas, receios e problemas vivenciados no cuidado em saúde $(n=45)$. Em um primeiro momento, todas as postagens foram organizadas da mais antiga para a mais recente, em seguida lidas na íntegra e categorizadas. O grupo que mais publica foi o com o menor número de usuários na ocasião da coleta (Grupo 1). Como os grupos compartilham as publicações uns dos outros, houve grande número de duplicatas $(\mathrm{n}=728)$.

Os grupos funcionam como um mural de notícias da imprensa tradicional. A maior parte das publicações era oriunda de sites de notícias e matérias de jornais $(n=254)$. Observou-se baixo engajamento, considerando o número reduzido de postagens contendo experiência pessoal ou familiar com ocorrência de incidentes ou eventos adversos no cuidado $(\mathrm{n}=45)$. Também se observou um número reduzido de curtidas, reações e compartilhamentos, em geral menor que 10 em 24 horas. Desses poucos comentários, foi identificado que, em geral, eram os mesmos usuários que comentavam as postagens. 
Verificou-se também o caráter informativo dos grupos que abordam direitos dos pacientes, caracterização do que é segurança do paciente, dos incidentes e eventos adversos entendidos pelos usuários como erros médicos e código de ética médica. Por outro lado, os grupos também apresentaram postagens relacionadas a propagandas, comentários sobre profissionais de saúde e pedidos de ajuda material ou financeira.

\section{Análise lexical com software Iramutec}

$\mathrm{Na}$ análise lexical, a divisão do corpus das postagens em segmentos de texto explorou a distribuição do vocabulário, por meio das classes lexicais, quantificando o texto e extraindo estruturas com significados ou temas mais representativos nas postagens de grupos de Facebook que abordavam a temática 'erros médicos'. Em seguida, na análise de similitude, apresentada no grafo, observa-se a ligação entre as palavras do corpus textual (Figura 1).

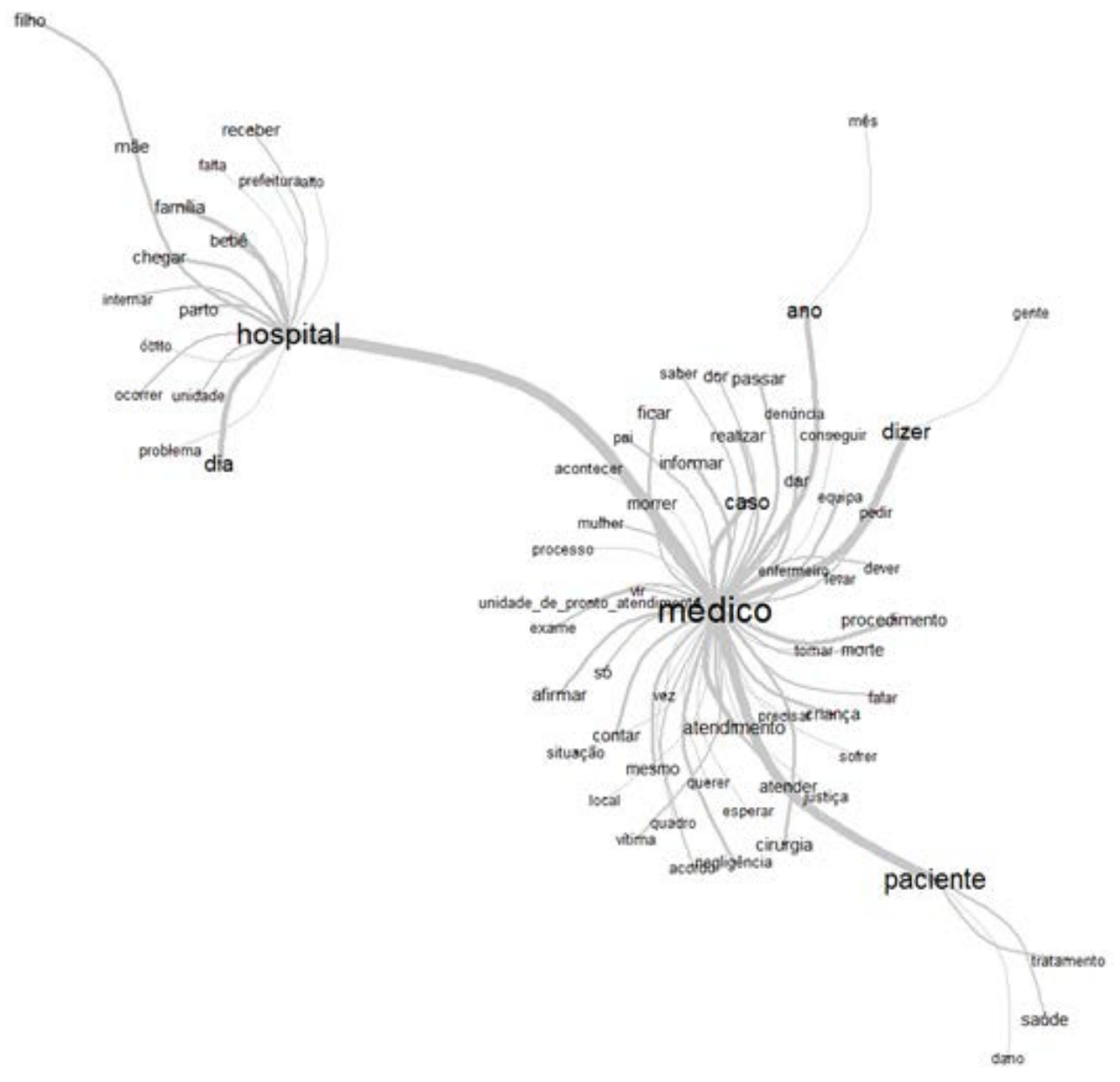

Figura 1 - Árvore de similitude dos grupos selecionados que abordaram a temática "erros médicos" no Facebook - até 100 ocorrências

Fonte: elaboração das autoras, utilizando software Iramutec.

O grupo com maior número de palavras teve como termo central a palavra 'médico', de onde ramificam outras palavras como: atendimento, procedimento, caso, negligência, dor e justiça. O segundo agrupamento de palavras derivado do primeiro teve 'hospital' como palavra central, e dela derivaram as palavras: 'unidade', 'chegar', 'internar', 'receber', 'parto', 'bebê', 'problema', 'óbito', 'prefeitura', 'dia', 'mãe' e 'filho'. Enquanto o terceiro grupo, que possuía o menor número de palavras, teve como centro a palavra 'paciente', ramificando nas palavras 'tratamento', 'saúde’ e 'dano'. Nesta configuração, também foi possível inferir o 
itinerário do cuidado, que teve como sujeito ativo o médico, foi realizado no hospital, sendo o paciente o sujeito que recebe o tratamento de saúde e pode sofrer dano.

\section{Análise de conteúdo das postagens do Facebook}

Emergiram quatro categorias: (i) incidentes e eventos adversos, (ii) fatores contribuintes, (iii) consequências dos eventos adversos, e (iv) reparação ou mitigação dos incidentes e eventos adversos.

\section{Incidentes e eventos adversos}

A primeira categoria identificada nas postagens dos grupos de Facebook foi 'incidentes e eventos adversos' reportados pelos pacientes e familiares nessa rede social. Contemplou uma ampla gama de questões relacionadas a atrasos no agendamento, cirurgia, erro no diagnóstico, comportamento dos profissionais e pacientes, medicamentos, infecções, procedimentos não cirúrgicos, realização de exames, queda, reações à transfusão de sangue e erro médico.

Quanto ao tipo de incidentes e eventos adversos identificados pelos pacientes e familiares, os resultados com base na análise desta categoria foram, no geral, semelhantes aos encontrados na literatura (HARRISON et al., 2015; VILLAR; DUARTE; MARTINS, 2020). No entanto, a frequência desses incidentes e eventos adversos apareceu em ordem distinta. Enquanto, nos estudos de revisão, os problemas relacionados a medicamentos despontaram como principais incidentes e eventos adversos (HARRISON et al., 2015; VILLAR; DUARTE; MARTINS, 2020), chamou a atenção o grande número de postagens relacionadas a processos administrativos indiretos relativos ao cuidado, que englobou atrasos no agendamento de consultas, exames, tratamento, diagnóstico e cirurgias.

A subcategoria 'processos administrativos indiretos relacionados ao cuidado', que contempla o atraso no diagnóstico ou tratamento, foi amplamente mencionada nas postagens. Nos casos de atraso no diagnóstico, em especial, a noção de dano dificilmente pode ser relacionada a novos problemas de saúde. Entretanto, os casos em que a deterioração do estado de saúde do paciente não é reconhecida e o tratamento não iniciado oportunamente no tempo têm sido descritos, na literatura, como problemas relacionados à segurança do paciente (BRADY et al., 2013). Os atrasos do tratamento acarretam agravamento do caso, necessidade de novos procedimentos e aumento do custo do tratamento (RODRIGUES et al., 2019). Os atrasos mencionados nas postagens contemplaram diversos tipos ou etapas do processo de cuidado, como atendimento de urgência ou emergência, ou marcação de consultas, exames e cirurgias.

"O médico só falou que não tinha material, que não teriam como fazer a cirurgia naquele dia e que remarcariam (...). Eu me preparei, fiz jejum e quando estava pronto para passar pela operação, me falaram de novo que não seria feita porque o material ainda estava em falta." (Postagem 29 - Reportagem).

A segunda subcategoria mencionada nas postagens foi relacionada à 'cirurgia'. Nas postagens, foram mencionados eventos adversos graves como retenção não intencional de corpo estranho em um paciente após a cirurgia, necessidade de nova cirurgia e óbito durante o procedimento ou imediatamente pós-operatório.

"Me lembro que o dia que, enfim, isso saiu de mim, tive uma dor que em muito se assemelha à dor de um parto. Foi um alívio, pois além da febre e tremor, eu sentia muita dor.”(Postagem 31 - Reportagem).

Revisão de estudos sobre casos cirúrgicos revelou ocorrência entre 5,8\% e 43,5\% de incidentes e eventos adversos após cirurgia (TEVIS; KENNEDY, 2013). No estudo realizado nos Estados Unidos da América (EUA) que analisou relatos de pacientes em pós-operatório emergiram três temas: dor e desconforto; riscos 
para segurança do paciente; melhor comunicação em relação às expectativas para cirurgia (RENDELL et al., 2020). Diferentemente dos estudos anteriores mencionados, as postagens relacionadas a incidentes e eventos adversos relacionados à cirurgia, nos grupos do Facebook, tratavam uma diversidade ampla de questões, desde problemas com agendamento até eventos de maior gravidade e óbitos.

A terceira subcategoria, que se refere a problemas relacionados ao 'procedimento e processo clínico', engloba problemas relacionados ao diagnóstico e a exames complementares, tratamento e procedimentos. Erro de diagnóstico foi o tema de maior frequência dentre as postagens relacionadas ao processo clínico.

"Durante um ano, o médico dizia que era infecção urinária [...]. Se tivesse descoberto um ano antes não tinha tirado próstata, bexiga, uma parte da uretra, uma parte do intestino." (Postagem 3 - Reportagem).

O processo diagnóstico ocorre ao longo do tempo e inclui fases de avaliação do histórico do paciente, exames, execução e interpretação de exames diagnósticos; acompanhamento e rastreamento de informações e exames; comunicação e coordenação do cuidado; e comportamento do paciente, adesão ao tratamento e engajamento. Erros de diagnóstico podem ocorrer em cada uma dessas fases, ou em mais de uma, e frequentemente refletem complexidades e vulnerabilidades do sistema de saúde (SINGH et al., 2017). Evidências sugerem que erros de diagnósticos representam um grande desafio, que a maioria das pessoas experimentará pelo menos um erro diagnóstico durante a vida (INSTITUTE OF MEDICINE, 2015). O resultado encontrado nas postagens foi condizente com a literatura. Estudo realizado nos EUA com 696 pacientes e familiares reportou que 30\% dos participantes identificaram um erro de diagnóstico ou no tratamento (SOUTHWICK; CRANLEY; HALLISY, 2015). Outro estudo encontrou cerca de 10 a $15 \%$ de erros de diagnósticos, em geral, no cuidado em saúde (GRABER, 2013). Revisão sistemática e meta-análise estimou prevalência de eventos adversos relacionados ao diagnóstico entre pacientes hospitalizados de $0,7 \%$, indicando que o erro de diagnóstico é um problema global. Contudo a baixa prevalência apontada provavelmente se deve ao local do estudo, ambiente hospitalar, uma vez que a maior parte dos erros de diagnósticos ocorre na atenção primária (SINGH et al., 2017; GUNDERSON et al., 2020).

O comportamento violento e abuso sexual também fizeram parte das postagens. Foram relatados problemas relacionados ao abuso sexual de profissionais contra pacientes no ambiente de cuidado. A violência sexual revela relações de poder complexas entre os sexos. $\mathrm{O}$ abuso sexual caracteriza-se como um problema de saúde pública, impactando física e emocionalmente as vítimas; as pessoas atingidas por esse tipo de violência são afetadas em sua autoimagem, autoestima e suas relações sociais (ANTONIO; FONTES, 2012). Esses casos tornam-se ainda mais graves quando o abuso sexual ocorre no ambiente de cuidado, onde as vítimas estão mais vulneráveis, dada a necessidade de buscar tratamento de saúde.

A violência física e verbal também fez parte da rotina dos serviços de saúde, segundo as postagens estudadas. A violência no local de trabalho pode ser um único evento ou uma série de pequenos incidentes recorrentes que, cumulativamente, têm o potencial de causar danos graves ao trabalhador e, por consequência, aos pacientes; ela pode ser física ou psicológica e tem como termos relacionados: assalto, ataque, abusos, bullying, assédio, assédio sexual, assédio racial e ameaças (WHO, 2002). A violência de pacientes e acompanhantes contra profissionais é um problema disseminado por todo o mundo. Como consequência, o compromisso com as boas práticas de cuidados e a confiança na própria capacidade profissional são reduzidos, podendo aumentar a ocorrência de erros e, em casos extremos, até mesmo de negligência médica (medical malpractice) (BERLANDA et al., 2019).

"[...] o adolescente ficou descontrolado e ameaçou agredir os servidores, entre eles, a médica. Foi contido, a segurança estava aqui, a polícia. Ele fez ameaças contra a médica que estava atendendo a familiar dele." (Postagem 25 - Reportagem). 
Diversos fatores afetam a possibilidade de ocorrer violência entre pacientes, acompanhantes e profissionais, sobretudo aqueles relacionados às pessoas como falhas na comunicação, informações deficientes, falta de confiança, expectativas não atingidas, insatisfação com o tratamento, discordância com o profissional, baixa adesão ao tratamento, perda de respeito pelos profissionais e percepção de um atendimento deficiente. A estes, somam-se fatores organizacionais e ambientais (BERLANDA et al., 2019).

Problemas relacionados à administração e ao uso de medicamentos constituem uma subcategoria de extrema importância para a segurança do paciente. Medicamentos foram identificados como principal preocupação de segurança reportada pelos pacientes (HARRISON et al., 2015; VILLAR; DUARTE; MARTINS, 2020). As reações adversas a medicamentos estão associadas à morbidade, aumento do tempo de permanência, aumento de custos para os sistemas de saúde e mortalidade em todo mundo (SHCHORY et al., 2020). Nas postagens, pacientes e familiares foram capazes de apontar problemas relacionados ao uso de medicamentos como problemas relacionados à administração e uso, troca de medicamentos, reação adversa, reação alérgica e não fornecimento de medicamentos. Em suma, essas postagens ilustram a capacidade que têm os usuários do grupo de identificar e comunicar esses problemas e explicitá-los.

"Conforme a decisão, a perícia detectou que a adolescente foi exposta a medicação sabidamente alergênica, e que isso já tinha sido comunicado pelo risco pré-operatório. Ou seja, por conta da aplicação do composto [...], houve reação alérgica (anafilaxia)." (Postagem 253 - Reportagem).

As infecções relacionadas à assistência à saúde (IRAS) também figuraram nas postagens. Elas constituem os eventos adversos mais frequentes ocorridos durante o atendimento, podendo se manifestar durante a internação ou após a alta hospitalar (WHO, 2011; CAVALCANTE et al., 2019). As IRAS constituem um grande problema para a segurança do paciente e, geralmente, acarretam gastos excessivos para o sistema de saúde e para as famílias, além de óbitos (INSTITUTE OF MEDICINE, 2000; CAVALCANTE et al., 2019).

"A paciente afirmou que, depois da cirurgia, contraiu uma micobactéria de crescimento rápido, que foi descoberta com análise de biópsia. Ela ainda disse que o hospital não disponibilizou nenhum infectologista para acompanhá-la, tendo somente recomendado que ela procurasse um hospital público para realizar o tratamento sem custo.”(Postagem 277 - Reportagem).

Com a análise das postagens, verificou-se que incidentes e eventos adversos são entendidos pelos usuários dos grupos do Facebook como ‘erro médico’. Essa expressão é compreendida como um conjunto de problemas na prática do cuidado em saúde, não especifico do médico, que podem ter diversas consequências danosas, inclusive o óbito. Para o Institute of Medicine, o erro médico é uma falha no planejamento ou execução da ação ou uso de um plano incorreto para atingir um objetivo (INSTITUTE OF MEDICINE, 200o). Já os pacientes o definem como um 'defeito’ no cuidado em saúde, e não um somatório de falhas ativas e latentes combinadas (REASON, 2000).

"O erro médico é um defeito na prestação do serviço de saúde que vem a causar dano ao paciente. Apesar do nome, ele não é cometido exclusivamente por um médico, pode decorrer da atuação de outros profissionais como enfermeiros, dentistas, nutricionistas ou até mesmo da administração do ambiente hospitalar.”(Postagem 38 - Participante).

Apesar de compreenderem que o "erro médico" pode ser cometido por outros profissionais, não consideraram atributos relativos à complexidade do caso ou à estrutura organizacional. Outros problemas relacionados ao cuidado que também surgiram nas postagens, porém com menor frequência, foram relacionados a procedimentos não cirúrgicos, exames laboratoriais, quedas e reações à transfusão de sangue. 


\section{Fatores contribuintes relacionados aos incidentes e aos eventos adversos}

Na segunda categoria - 'fatores contribuintes'-foramidentificados nas postagens problemas relacionados ao ambiente e à estrutura, comunicação, identificação do paciente e negligência. Problemas relacionados à estrutura podem causar desde desconforto no paciente, atraso na realização de procedimentos, realização de novo procedimento sem necessidade, infecções e até óbitos.

"O funcionário explica que a falta de refrigeração adequada também pode levar à proliferação de bactéria na UTI. 'A gente deixa a porta aberta para circular [o ar]. A janela fica aberta. Lá [nos leitos] não pode deixar a janela tão aberta por causa de mosquito e mosca." (Postagem 266 - Reportagem).

Outra preocupação que apareceu com frequência foram questões de comunicação. Entre elas, destacaram-se problemas na passagem de plantão; o fato de o profissional não ter informado ao paciente os riscos de uma cirurgia ou não tratar o paciente com dignidade e respeito.

"Segundo a dona de casa, ela chegou ao local em uma troca de plantões. Um médico pediu que a criança fosse internada, mas não teria avisado ao profissional que ficaria no lugar dele. Este, por sua vez, deu alta à criança, que apresentou os mesmos sintomas no dia seguinte." (Postagem 139 - Reportagem).

Outras questões contemplaram a guarda de documentação e o fluxo de informação. Atraso ou recusa na entrega de prontuário emergiram nas postagens; apesar do direito do paciente a ver as informações registradas, seu sigilo deve ser garantido (ARAÚJO; RECHMANN; MAGALHÃES, 2019).

Problemas relacionados à comunicação também foram amplamente identificados na literatura como fatores contribuintes para incidentes e eventos adversos reconhecidos pelos pacientes. Estudo multicêntrico, com pacientes internados na Inglaterra, reportou preocupações com a comunicação entre profissionais e pacientes, aliado ao número insuficiente de profissionais, e ao esquecimento de tarefas; relatou também outras questões relacionadas ao ambiente e à estrutura, incluindo iluminação, barulho, limpeza e falta ou mal funcionamento de equipamentos (O'HARA et al., 2018).

Outro fator desencadeante dos incidentes e eventos adversos, identificado nas postagens, foi a negligência do cuidado, entendida como falha de um cuidador designado para atender às necessidades de alguém que não tenha independência. Embora a 'negligência em relação ao paciente' seja uma expressão amplamente usada pelo público e pela mídia para descrever o atendimento precário, seu significado legal depende do contexto sociocultural, portanto requer precisão. Reader e Gillepsie (2013) reconceituaram a expressão 'negligência em relação ao paciente' como 'negligência do cuidado', referindo-se a falhas no cuidado em saúde que ultrapassam o limite esperado, mas são incapazes de causar dano imediato, embora levem pacientes e familiares a acreditarem que os profissionais não estão preocupados com o bem-estar do paciente (READER; GILLESPIE, 2013).

\section{Consequências dos eventos adversos}

Na categoria 'consequências dos eventos adversos' destacaram-se problemas emocionais, incapacidade temporária ou permanente e óbitos.

"Além das dores, físicas e emocionais, ficou também uma grande cicatriz que eu tive que cobrir com uma tatuagem, para que me olhar em um espelho não fosse algo tão difícil." (Postagem 31 - Reportagem). 
Os resultados foram semelhantes aos apontados na literatura que destacaram impactos de curto ou longo prazo para pacientes e familiares, tais como: (i) psicológicos, como raiva, autoculpabilização, medo, perda da confiança no cuidado em saúde, memórias vívidas do evento, luto, traumas, depressão, ideação suicida; efeitos sociais/comportamentais, necessidade de compartilhar a experiência, mudança da visão de si mesmo, mudanças de comportamento na busca dos serviços de saúde; (ii) físicos, como disfunção ou perda de membros, órgãos ou sentidos, necessidade de novas cirurgias, tratamentos, reabilitação, terapia e novos medicamentos para lidar com a nova doença ou condição; (iii) financeiros, como perda do emprego, seguro-saúde, acúmulo de dívidas com contas do tratamento de saúde; (iv) suicídios e óbitos (SOUTHWICK; CRANLEY; HALLISY, 2015; MIRA et al., 2017; OTTOSEN et al., 2018). Para os familiares destacam-se: trauma emocional, estresse de cuidar do paciente, perda financeira, do estilo de vida, do emprego, da moradia, culpa e divórcio (SOUTHWICK; CRANLEY; HALLISY, 2015).

\title{
Reparação ou mitigação dos eventos adversos
}

Nesta última categoria, pacientes e familiares buscavam justiça, seja divina ou judicial para reparação de danos morais e materiais advindos de problemas vivenciados no cuidado em saúde. As postagens sublinhando desejo ou ação de reparação listaram problemas ligados à cirurgia, obstetrícia, atrasos no atendimento, erro de diagnóstico, medicamentos, tratamento inadequado, assédio, paciente adquiriu outra doença após o tratamento e óbito.

\begin{abstract}
"Inclusive minha sobrinha veio pra cá [...] para não correr o risco de ter de fazer o parto no carro. Ela não teve seu bebê no domingo [...] por descaso médico, negligência médica. Deixaram o bebê morrer na barriga da mãe" [...]. "Nós queremos que seja feita justiça, e é isso que vamos cobrar, é para isso que vamos lutar." (Postagem 89 - Reportagem).
\end{abstract}

Achados semelhantes são encontrados na literatura nacional (BRAGA; ERTLER; GARBIN, 2017; GOMES; SÁ, 2017; CARVALHO; CADIDÉ, 2019) e internacional (GIRALDO et al., 2016; HWANG et al., 2018). No Brasil, a judicialização engloba serviços ambulatoriais e cirúrgicos (CARVALHO; CADIDÉ, 2019). A maior parte dos processos refere-se a danos morais e materiais, ligados às especialidades de ginecologia e obstetrícia e a cirurgias, e o réu foi o setor privado (GOMES; SÁ, 2017).

Nos estudos internacionais, o maior motivo foi relacionado ao diagnóstico, como falhas, atrasos ou erros (GIRALDO et al., 2016). Contudo, outro estudo, revelou que os problemas mais frequentes nas alegações judiciais foram relacionados à cirurgia e a complicações perinatais, sendo que as três especialidades mais arroladas nas alegações judiciais foram obstetrícia, ortopedia e medicina de emergência (HWANG et al., 2018).

\section{CONSIDERAÇÕES FINAIS}

Nos últimos anos, a internet surgiu como campo de delimitação de novas fronteiras sociais e culturais. O Facebook destaca-se como campo de pesquisa na saúde, devido à sua conformação e à possibilidade de criação de grupos e comunidades abrangendo usuários com objetivos em comum.

Este estudo apresenta limites dos quais vale mencionar, em primeiro lugar, a incipiência do tema segurança do paciente no contexto brasileiro que, certamente, influencia a percepção dos pacientes acerca da identificação desses problemas. Inicialmente, considerou-se adotar a ferramenta Netvizz para extração de posts do Facebook; no entanto, ao longo do planejamento deste estudo, a ferramenta foi descontinuada e toda a coleta precisou ser feita manualmente. No entanto, o desafio de coletar de forma manual um conteúdo tão dinâmico como o de uma rede social permitiu assegurar que os preceitos éticos fossem respeitados, 
além de possibilitar uma imersão em profundidade nesse conteúdo. Assim, apenas grupos abertos foram mapeados, não foi possível captar informações de grupos fechados e dimensionar diferença entre eles.

Dentro dos grupos estudados, os usuários compartilharam preocupações com a qualidade do cuidado e segurança do paciente, assumindo a denominação central de erros médicos. O conteúdo postado pelos usuários dos grupos estudados abordou incidentes e eventos adversos vivenciados relacionados a medicamentos, infecções e cirurgias. Como fatores contribuintes da ocorrência de incidentes e eventos adversos emergiram preocupações relacionadas à comunicação e à estrutura. Também foram destacadas as consequências desses eventos adversos e as ações requerendo reparação e mitigações destes, o que denota o potencial dos grupos como espaço de esclarecimento, debate, denúncia, pesquisa e divulgação de conteúdo voltado para segurança e qualidade no cuidado em saúde.

Na visão dos usuários dos grupos do Facebook, incidentes e eventos adversos foram descritos, de modo geral, como erros médicos. Apesar dessa forte expressão adotada e de sua relevância para pacientes e familiares que vivenciaram incidentes e eventos adversos, nos grupos analisados, foi encontrado volume reduzido de postagens de autoria própria dos usuários. Além disso, o conteúdo foi pouco compartilhado, o que pode indicar que esses grupos funcionam mais como um mural de notícias da mídia tradicional do que como espaços de compartilhamentos de suas preocupações e experiências no cuidado de sua saúde.

Em suma, pesquisas que exploram o material produzido e compartilhado por usuários em mídias sociais, oferecem um rico material que aponta contextos e narrativas diversas sobre a saúde e o cuidado em saúde. Esta pesquisa mostrou que os usuários do Facebook foram capazes de identificar e discutir diversas questões relacionadas à segurança do paciente e à qualidade do cuidado, embora não utilizem esses termos nos relatos postados. Além disso, nas postagens foi possível identificar o desejo e a busca de reparação e mitigação de incidentes e eventos adversos no cenário nacional.

Contudo, para que a perspectiva do usuário impacte as práticas dos serviços de saúde, para além da divulgação e judicialização dos processos, é importante que novas estratégias de divulgação do conhecimento via plataformas de mídia digital como o Facebook sejam adotadas, visto que se mostraram capazes de aumentar o engajamento e o empoderamento dos usuários no cuidado em saúde.

\section{REFERÊNCIAS}

ANTONIO, Eliana Maria Restum; FONTES, Tereza Maria Pereira. Bioética e aspectos epidemiológicos de vítimas de violência sexual em hospital maternidade. Revista Bioética, Brasília, DF, v. 20, n. 2, p. 280-287, 2012. Disponível em: https://revistabioetica.cfm.org.br/index.php/revista_bioetica/article/view/746/777. Acesso em: 04 nov. 2020.

ARAÚJO, Ana Thereza Meireles; RECHMANN, Itanaina Lemos; MAGALHÃES, Thayná Andrade. O sigilo do prontuário médico como um direito essencial do paciente: uma análise a partir das normativas do Conselho Federal de Medicina. Cadernos Ibero-Americanos de Direito Sanitário, Brasília, DF, v. 8, n. 1, jan./mar. 2019, p. 95-109. DOI: http://dx.doi.org/10.17566/ciads.v8i1.517. Disponível em: https://www.cadernos. prodisa.fiocruz.br/index.php/cadernos/article/view/517/575. Acesso em: 08 jun. 2021.

BARDIN, Laurence. Análise de conteúdo. 5. ed. Portugal: Edições 70, 2011.

BERLANDA, Sabrina et al. Addressing risks of violence against healthcare staff in emergency departments: the effects of job satisfaction and attachment style. BioMed Research International, Nova York, v. 2019, p. 1-12, 28 maio 2019. DOI: https://doi.org/10.1155/2019/5430870. Disponível em: https://www.ncbi.nlm.nih.gov/ pmc/articles/PMC6558649/pdf/BMRI2019-5430870.pdf. Acesso em: 04 nov. 2020.

BRADY, Patrick W. et al. Improving situation awareness to reduce unrecognized clinical deterioration and serious safety events. Pediatrics, Elk Grove, v. 131, n. 1, p. e298-e308, jan. 2013. DOI: https://doi. org/10.1542/peds.2012-1364. Disponível em: https://www.ncbi.nlm.nih.gov/pmc/articles/PMC4528338/pdf/ peds.2012-1364.pdf. Acesso em: 04 nov. 2020. 
BRAGA, Isabel de Fátima Alvim; ERTLER, Laila Zelkcovicz; GARBIN, Helena Beatriz da Rocha. Entendimento do Tribunal de Justiça do Pará sobre o erro médico na esfera penal. ABCS Health Sciences, Santo André, v. 42, n. 3, dez. 2017, p. 156-160. DOI: http://dx.doi.org/10.7322/abcshs.v42i3.987. Disponível em: https:// docs.bvsalud.org/biblioref/2017/12/876230/42abcs156.pdf. Acesso em: 04 nov. 2020.

CAMARGO, Brigido Vizeu; JUSTO, Ana Maria. IRAMUTEQ: Um software gratuito para análise de dados textuais. Temas em Psicologia, Ribeirão Preto, v. 21, n. 2, p. 513-518, 2013. DOI: http://dx.doi.org/10.9788/ TP2013.2-16. Disponível em: http://pepsic.bvsalud.org/scielo.php?script=sci arttext\&pid=S1413389X2013000200016. Acesso em: 04 nov. 2020.

CARVALHO, Regina Ribeiro Parizi; CADIDÉ, Welington Cardoso de Oliveira. Riscos legais litigiosos na assistência à saúde. Revista de Enfermagem UFPE on line, Recife, v. 13, p. e242297, out. 2019. DOI: http://dx.doi.org/10.5205/1981-8963.2019.242297. Disponível em: https://periodicos.ufpe.br/revistas/ revistaenfermagem/article/view/242297/33742. Acesso em: 04 nov. 2020.

CAVALCANTE, Elisângela Franco de Oliveira et al. Implementação dos núcleos de segurança do paciente e as infecções relacionadas à assistência à saúde. Revista Gaúcha de Enfermagem, Porto Alegre, v. 40, n. spe, p. e20180306, 2019. DOI: https://doi.org/10.1590/1983-1447.2019.20180306. Disponível em: https:// www.scielo.br/j/rgenf/a/XnshRsYTrr4dQKSnkznwDYw/?lang=pt. Acesso em: 04 nov. 2020.

DESLANDES, Suely; COUTINHO, Tiago. Pesquisa social em ambientes digitais em tempos de COVID-19: notas teórico-metodológicas. Cadernos de Saúde Pública, Rio de Janeiro, v. 36, n. 11, p. e00223120, 2020. DOI: https://doi.org/10.1590/0102-311X00223120. Disponível em: https://www.scielo.br/j/csp/a/hz9h4Fm4 mdrvnZwTfKRpRNq/?lang=pt. Acesso em: $16 \mathrm{dez} .2020$.

FRANZ, Daschel et al. Using Facebook for qualitative research: a brief primer. Journal of Medical Internet Research, Toronto, v. 21, n. 8, e13544, 2019. DOI: https://dx.doi.org/10.2196\%2F13544. Disponível: https:// www.ncbi.nlm.nih.gov/pmc/articles/PMC6711038/. Acesso em: 8 jun. 2021.

GIRALDO, Priscila et al. Eleven-year descriptive analysis of closed court verdicts on medical errors in Spain and Massachusetts. BMJ Open, Londres, v. 6, n. 8, p. e011644, ago. 2016. DOI: https://dx.doi.org/10.1136\% 2Fbmjopen-2016-011644. Disponível em: https://www.ncbi.nlm.nih.gov/pmc/articles/PMC5013385/. Acesso em: 04 nov. 2020.

GOMES, Talita Rodrigues; SÁ, Maria Célia Delduque Nogueira Pires de. O erro médico sob o olhar do Judiciário: uma investigação no Tribunal de Justiça do Distrito Federal e Territórios. Cadernos IberoAmericanos de Direito Sanitário, Brasília, DF, v. 6, n. 1, mar. 2017, p. 72-85. DOI: https://doi.org/10.17566/ ciads.v6i1.362. Disponível em: https://www.cadernos.prodisa.fiocruz.br/index.php/cadernos/article/ view/362/446. Acesso em: 07 jun. 2020.

GRABER, Mark L. The incidence of diagnostic error in medicine. BMJ Quality \& Safety, Londres, v. 22, n. suppl 2, p. ii21-ii27, out. 2013. DOI: https://doi.org/10.1136/bmjgs-2012-001615. Disponível em: https://www. ncbi.nlm.nih.gov/pmc/articles/PMC3786666/pdf/bmjqs-2012-001615.pdf. Acesso em: 04 nov. 2020.

GREAVES, Felix et al. Harnessing the cloud of patient experience: using social media to detect poor quality healthcare. BMJ Quality and Safety, Londres, v. 22, n. 3, p. 251-255, 2013. DOI: http://dx.doi.org/10.1136/ bmjas-2012-001527. Disponível em: https://qualitysafety.bmj.com/content/22/3/251. Acesso em 24 jun. 2019.

GUNDERSON, Craig G. et al. Prevalence of harmful diagnostic errors in hospitalised adults: a systematic review and meta-analysis. BMJ Quality \& Safety, Londres, v. 29, n. 12, p.1008-1018, abr. 2020. DOI: http:// dx.doi.org/10.1136/bmjqs-2019-010822. Disponível em: https://qualitysafety.bmj.com/content/29/12/1008. Acesso em: 04 nov. 2020.

HARRISON, Reema et al. The missing evidence: a systematic review of patients' experiences of adverse events in health care. International Journal for Quality in Health Care, Oxford, v. 27, n. 6, p. 424-442, dez. 2015. DOI: https://doi.org/10.1093/intqhc/mzv075. Disponível em: https://academic.oup.com/intqhc/ article/27/6/424/2357399. Acesso em: 13 jul. 2018.

HUERTA, Timothy R. et al. Patient safety errors: leveraging health information technology to facilitate patient reporting. Journal for Healthcare Quality, Skokie, v. 38, n. 1, p.17-23, 2016. DOI: https://doi.org/10.1097/ ¡hq.0000000000000022. Disponível em: https://journals.Iww.com/jhqonline/Abstract/2016/01000/Patient Safety Errors Leveraging Health.3.aspx. Acesso em 30 set. 2020. 
HWANG, Chi-Yuan et al. A 12-year analysis of closed medical malpractice claims of the Taiwan civil court: a retrospective study. Medicine, Hagerstown, v. 97, n. 13, p. e0237, mar. 2018. DOI: https://dx.doi.org/10.109 7\%2FMD.0000000000010237. Disponível em: https://www.ncbi.nlm.nih.gov/pmc/articles/PMC5895413/. Acesso em: 04 nov. 2020.

INSTITUTE OF MEDICINE. To err is human: building a safer health system. Washington, D.C: National Academies Press, 2000.

INSTITUTE OF MEDICINE. Improving diagnosis in health care. Washington, DC: National Academies of Sciences, Engineering, and Medicine, 2015.

MIRA, Jose Joaquin et al. Lessons learned for reducing the negative impact of adverse events on patients, health professionals and healthcare organizations. International Journal for Quality in Health Care, Oxford, v. 29, n. 4, p. 450-460, ago. 2017. DOI: https://doi.org/10.1093/intqhc/mzx056. Disponível em: https:// pubmed.ncbi.nlm.nih.gov/28934401/. Acesso em: 04 nov. 2020.

MIRANDA, Fernanda Santana; ROCHA, Dais Gonçalves. O uso do Facebook na promoção da saúde: uma revisão bibliográfica sobre empoderamento e participação popular. Revista Eletrônica de Comunicação, Informação e Inovação em Saúde, Rio de Janeiro, v. 12, n. 2, abr./jun. 2018, p. 232-243. DOI: https://doi. org/10.29397/reciis.v12i2.1331. Disponível em: https://www.reciis.icict.fiocruz.br/index.php/reciis/article/ view/1331. Acesso em: 07 jun. 2021.

NASCIMENTO, Adriano Roberto Afonso do; MENANDRO, Paulo Rogério Meira. Análise lexical e análise de conteúdo: uma proposta de utilização conjugada. Estudos e Pesquisa em Psicologia, Rio de Janeiro, v. 6, n. 2, p. 72-88, 2006. Disponível em: http://www.revispsi.uerj.br/v6n2/artigos/pdf/v6n2a07.pdf. Acesso em: 18 jan. 2021.

O'HARA, Jane K. et al. What can patients tell us about the quality and safety of hospital care? Findings from a UK multicentre survey study. BMJ Quality \& Safety, Londres, v. 27, n. 9, set. 2018, p. 673-682. DOI: https://doi.org/10.1136/bmjgs-2017-006974. Disponível em: https://qualitysafety.bmj.com/content/ghc/ early/2018/03/15/bmjqs-2017-006974.full.pdf. Acesso em: 4 nov. 2020.

OTTOSEN, Madelene J. et al. Long-term impacts faced by patients and families after harmful healthcare events. Journal of Patient Safety, Filadélfia, v. 17, n. 8, p. e1145-e1151, dez. 2021. DOI: https://doi. org/10.1097/pts.0000000000000451. Disponível em: https://www.ncbi.nlm.nih.gov/pmc/articles/ PMC6050155/. Acesso em: 06 dez. 2021.

READER, Tom W.; GILLESPIE, Alex. Patient neglect in healthcare institutions: a systematic review and conceptual model. BMC Health Services Research, Londres, v. 13, n. 1, dez. 2013, p. 1-15. DOI: https:// dx.doi.org/10.1186\%2F1472-6963-13-156. Disponível em: https://www.ncbi.nlm.nih.gov/pmc/articles/ PMC3660245/. Acesso em: 04 nov. 2020.

REASON, James. Human error: models and management. BMJ, Londres, v. 320, p. 768-770, 2000. DOI: https://doi.org/10.1136/bmj.320.7237.768. Disponível em: https://www.bmj.com/content/320/7237/768. Acesso em: 16 out. 2020.

RENDELL, Victoria R. et al. Severity of postoperative complications from the perspective of the patient. Journal of Patient Experience, Cleveland, v. 7, n. 6, p.1568-1576, dez. 2020. DOI: https://doi.org/10.1177/2374373519893199. Disponível em: https://journals.sagepub.com/doi/ full/10.1177/2374373519893199. Acesso em: 04 nov. 2020.

RODRIGUES, Thaynara Pereira et al. Ocorrência de eventos adversos em unidade de hemodinâmica. Revista de Enfermagem UFPE On line, Recife, v. 13, n. 1, p. 86-95, jan. 2019. DOI: http://dx.doi.org/10.5205/19818963-v13i1a235853p86-95-2019. Disponível em: https://periodicos.ufpe.br/revistas/revistaenfermagem/ article/view/235853/31131. Acesso em: 04 nov. 2020.

ROZENBLUM, Ronen; BATES, David. The role of patient-facing technologies to empower patients and improve safety. AHRQ Patient Safety Network, Rockville, 1 nov. 2021. Perspectives. Disponível em: https://psnet. ahrq.gov/perspective/role-patient-facing-technologies-empower-patients-and-improve-safety. Acesso em: 02 dez. 2021

ROZENBLUM, Ronen; BATES, David. Patient-centred healthcare, social media and the internet: the perfect storm? BMJ Quality \& Safety, Londres, v. 22, n. 3, p. 183-186, mar. 2013. DOI: http://dx.doi. org/10.1136/bmiqs-2012-001744. Disponível em: https://qualitysafety.bmj.com/content/22/3/183. Acesso em: 16 dez. 2020. 
SHCHORY, Miri Potlog et al. Increasing adverse drug reaction reporting: how can we do better? PLoS One, São Francisco, v. 15, n. 8, ago. 2020, p.1-15. DOI: https://doi.org/10.1371/journal.pone.0235591. Disponível em: https://journals.plos.org/plosone/article?id=10.1371/journal.pone.0235591. Acesso em: 04 nov. 2020.

SINGH, Hardeep et al. The global burden of diagnostic errors in primary care. BMJ Quality \& Safety, Londres, v. 26, n. 6, p. 484-494, 2017. DOI: http://dx.doi.org/10.1136/bmjqs-2016-005401. Disponível em: https:// qualitysafety.bmj.com/content/26/6/484. Acesso em: 08 jun. 2021.

SOUTHWICK, Frederick S.; CRANLEY, Nicole M.; HALLISY, Julia A. A patient-initiated voluntary online survey of adverse medical events: the perspective of 696 injured patients and families. BMJ Quality \& Safety, Londres, v. 24, n. 10, p. 620-629, out. 2015. DOI: https://doi.org/10.1136/bmjqs-2015-003980. Disponível em: https://qualitysafety.bmj.com/content/qhc/24/10/620.full.pdf. Acesso em: 22 set. 2020.

STATISTA RESEARCH DEPARTMENT. Number of internet users in Brazil from 2017 to 2026. [S. I.]: Statista, 2021. Disponível em: https://www.statista.com/statistics/255208/number-of-internet-users-inbrazill. Acesso em: 18 jan. 2021.

TEVIS, Sarah E.; KENNEDY, Gregory D. Postoperative complications and implications on patient-centered outcomes. Journal of Surgical Research, Nova York, v. 181, n. 1, p. 106-113, maio 2013. DOI: https:// dx.doi.org/10.1016\%2Fi.jss.2013.01.032. Disponível em: https://www.ncbi.nlm.nih.gov/pmc/articles/ PMC3637983/. Acesso em 4 nov. 2020.

VAN DE BELT, Tom H. et al. Using patient experiences on Dutch social media to supervise health care services: exploratory study. Journal of Medical Internet Research, Toronto, v. 17, n. 1, p. e7, jan. 2015. DOI: https://dx.doi.org/10.2196\%2Fimir.3906. Disponível em: https://www.ncbi.nlm.nih.gov/pmc/articles/ PMC4319082l. Acesso em 30 set. 2020.

VILLAR, Vanessa Cristina Felippe Lopes; DUARTE, Sabrina da Costa Machado; MARTINS, Mônica. Segurança do paciente no cuidado hospitalar: uma revisão sobre a perspectiva do paciente. Cadernos de Saúde Pública, Rio de Janeiro, v. 36, n. 12, p. 1-20, dez. 2020. DOI: https://doi.org/10.1590/0102311X00223019. Disponível em: https://www.scielo.br/j/csp/a/Yj4QxnXJJxJbsVhrrrCQwQr/?lang=pt. Acesso em: 18 jan. 2021.

WORLD HEALTH ORGANIZATION (WHO). Conceptual framework for the international classification for patient safety: final technical report January 2009. Genebra: World Health Organization, 2009.

WORLD HEALTH ORGANIZATION (WHO). Framework guidelines for addressing workplace violence in the health sector. Genebra: World Health Organization, 2002. Disponível em: https://www.who.int/ publications/i/item/9221134466. Acesso em: $11 \mathrm{dez} .2021$.

WORLD HEALTH ORGANIZATION (WHO). Patients for patient safety: partnership for safer health care. Genebra: World Health Organization, 2013. Disponivel em: https://www.who.int/patientsafety/patients for patient/PFPS_brochure_2013.pdf. Acesso em 4 nov. 2020.

WORLD HEALTH ORGANIZATION (WHO). Report on the burden of endemic health care associated infection worldwide. Geneva: World Health Organization, 2011. Disponível em: https://apps.who.int/iris/bitstream/handle/10665/80135/9789241501507_eng. pdf;jsessionid=8ACFE86B600E4E0670BA1D901C8DA699? sequence=1. Acesso em: 04 nov. 2020.

WORLD HEALTH ORGANIZATION (WHO). World Patient Safety Day 2019. Genebra: World Health Organization, c2021. Disponível em: https://www.who.int/campaigns/world-patient-safety-day/2019. Acesso em 15 dez. 2020. 